\title{
Hip aspiration culture: analysing data from a single operator series investigating periprosthetic joint infection
}

\author{
Connor J. Barker ${ }^{1}$, Alan Marriot ${ }^{2}$, Munir Khan ${ }^{3}$, Tamsin Oswald ${ }^{2}$, Samuel J. Tingle ${ }^{4}$, \\ Paul F. Partington ${ }^{2}$, Ian Carluke ${ }^{2}$, and Mike R. Reed $^{2}$ \\ ${ }^{1}$ South Tees NHS Foundation Trust, Middlesbrough, TS4 3BW, UK \\ ${ }^{2}$ Northumbria Healthcare NHS Foundation Trust, Cramlington, NE23 6NZ, UK \\ ${ }^{3}$ Northumbria Healthcare NHS Foundation trust, Cramlington, NE23 6NZ, UK \\ ${ }^{4}$ Newcastle upon Tyne Hospitals NHS Foundation Trust, Freeman Hospital, \\ Newcastle upon Tyne, NE7 7DN, UK
}

Correspondence: Connor J. Barker (connor.barker@nhs.net)

Received: 7 October 2020 - Revised: 14 February 2021 - Accepted: 9 April 2021 - Published: 10 May 2021

\begin{abstract}
Introduction: We undertook this study to know the sensitivity, specificity and post-test probabilities of hip aspiration when diagnosing periprosthetic hip infections. We also examined "dry tap" (injection with saline and aspiration) results and aspiration volumes. Methods: This is a retrospective cohort study of patients aspirated for suspected periprosthetic joint infection between July 2012 and October 2016. All aspirations were carried out by one trained surgical care practitioner (SCP). All aspirations followed an aseptic technique and fluoroscopic guidance. Aspiration was compared to tissue biopsy taken at revision. Aspiration volumes were analysed for comparison. Results: Between January 2012 and September 2016, 461 hip aspirations were performed by our SCP. Of these 125 progressed to revision. We calculated sensitivity $59 \%$ (confidence interval (CI) $35 \%-82 \%$ ) and specificity $94 \%$ (CI $89 \%-98 \%$ ). Pre-test probability for our cohort was 0.14 . Positive post-test probability was 0.59 and negative post-test probability 0.06 . Aspiration volume for infected $(n=17)$ and non-infected $(n=$ 108 ) joints was compared and showed no significant difference. Dry taps were experienced five times; in each instance the dry tap agreed with the biopsy result. Conclusions: Our data show that hip aspiration culture is a highly specific investigation for diagnosing infection but that it is not sensitive. Aspiration volume showed no significant difference between infected and non-infected groups. Each time a joint was infiltrated with saline to achieve a result, the result matched tissue sampling.
\end{abstract}

\section{Introduction}

Periprosthetic joint infection (PJI) is a painful condition that results in early failure of arthroplasty and significant cost (Peel et al., 2013; Cross et al., 2014). Infection burden for all hip replacements in England is reported to be $1.6 \%$ when there is high-quality post-discharge surveillance (Tanner et al., 2013). There is no one gold standard test for PJI: the difficulty in defining it has been much discussed (Parvizi et al., 2011, 2013) and has led to the development of multi-test diagnostic criteria (Parvizi et al., 2018). Diagnoses are currently informed by several factors such as the clinical picture, pre-operative indicators such as blood tests (C-reactive protein, CRP, and erythrocyte sedimentation rate, ESR) and synovial fluid tests (Parvizi et al., 2018). CRP and ESR are sensitive but not specific (Piper et al., 2010), whereas synovial fluid aspiration is an invasive test with operator-dependent success rates (Tingle et al., 2016). In our institution the majority of hip aspirations for suspected PJI are carried out by a single trained orthopaedic surgical care practitioner (SCP). This approach has been proven to lower the rate of dry tap aspirations (Tingle et al., 2016). When unable to obtain a synovial aspirate, the joint was injected with saline and reaspirated immediately. This method of injecting with saline 
is not often reported in the literature (Partridge et al., 2018). The primary aim of this study is to assess the utility of joint aspiration from the viewpoint of an accurate and reliable dataset provided by a single operator. This will be done by analysing aspiration culture post-test probabilities, sensitivity and specificity, which are then compared to common serological markers. Secondary measures include comparing aspirate volumes of PJI versus aseptic joints, comparing the use of blood culture bottles versus universal containers and the results of dry tap aspirations.

\section{Methods}

We have analysed all hip aspirations performed for suspected PJI by our SCP between July 2012 and October 2016. In this period, 461 aspirations were performed, of which 107 patients progressed to revision. Patients were included in the study if they had a hip aspiration for suspected PJI and had progressed to revision, and patients were excluded if they had not progressed to revision. The cohort of 107 patients experienced 125 of the 461 aspirations. The larger number of aspirations than patients can be accounted for by 14 repeat aspirations and 4 bilateral aspirations. Of the 125 aspirations, 60 were performed on males and 65 on females, and mean age at time of aspiration was 66 (38-94) and 73 (54-98) respectively. All aspirations were fluoroscopy guided and followed an aseptic technique, including skin preparation with a $2 \%$ chlorhexidine wash. The number of patients receiving antibiotics at the time of aspiration is unknown. All aspirations were performed in a surgical theatre; a theatre with laminar airflow was sometimes but not always available. If an aspiration was dry, a fluid washout was performed by injecting into the joint $10 \mathrm{~mL}$ normal saline and immediately re-aspirating $(n=5)$. Contrast media were not used to confirm the source of the aspirate. Specimens were put into an anaerobic blood culture bottle, an aerobic culture bottle and a sterile universal container, all of which were manufactured by Becton Dickinson. At revision, five different tissue specimens were taken with separate sterile instruments (Atkins et al., 1998). Prior to procedure CRP and ESR were obtained in 117 of 125 cases.

\section{Microbiology}

UK protocols for investigation of orthopaedic-implantassociated infections were followed as a minimum standard throughout (Public Health England, 2016). Blood culture bottles were incubated for $10 \mathrm{~d}$ and plated out if identified as positive. Where the clinical picture is consistent with PJI but no growth after $10 \mathrm{~d}$, broth cultures were incubated for $14 \mathrm{~d}$ as per national procedure (Public Health England, 2016). Aspirates in sterile universal containers are plated out and plates read at either 48 or $72 \mathrm{~h}$, and then an anaerobic plate is read at $5 \mathrm{~d}$. Five drops are put in enrichment broth and plated out when cloudy or at $5 \mathrm{~d}$; these plates are then read at $48 \mathrm{~h}$. If a fungal infection is suspected, a Sabouraud agar plate was used. Tissue was homogenised in $1 \mathrm{~mL}$ of sterile saline by glass beads and applied to a vortex for $15 \mathrm{~s}$. The following media were inoculated with each specimen: Robertson's cooked meat broth, chocolate blood agar, blood agar, fastidious anaerobe agar (FAA) $+5 \mu \mathrm{g}$ metronidazole (one cultivated for $3 \mathrm{~d}$ and another for $5 \mathrm{~d}$ ), and chromogenic UTI media. A single specimen from the entire series was sent for tuberculosis culture. Biopsy results were considered to be infected if three or more of the samples grew the same organism; this was our control test. If two or fewer samples grew the same organism, the result was considered to be not infected. This is an approach proven to be most likely to predict infection from biopsy (Atkins et al., 1998). By definition there were no false positives or false negatives in the tissue sample results. Cut-offs for CRP and ESR were $>10.3 \mathrm{mg} / \mathrm{L}$ and $>13 \mathrm{~mm} / \mathrm{h}$ respectively, as recommended by meta-analysis (Piper et al., 2010).

When calculating confidence intervals (CIs), an alpha level of 0.05 was used to calculate $95 \%$ CI. Analysis of aspiration volume data was performed using RStudio version 1.1.383 (RStudio, 2015). Aspirations from patients with an infection (confirmed by biopsy) were compared to those confirmed non-infected. A Shapiro-Wilk normality test was performed, followed by a Mann-Whitney test to compare the distribution of aspiration volume for infected and non-infected patients.

\section{Results}

\subsection{Aspiration culture}

The results of our statistical analysis are presented in Table 1; 125 aspiration cultures with subsequent tissue samples were available for analysis. For our population of patients aspirated for suspected PJI, we calculated a pre-test probability of infection to be 0.14 . Aspiration culture positive post-test probability and negative post-test probability were 0.59 and 0.06 respectively. At time of tissue sampling, the number of procedures where the patient was on antibiotics at the time of revision was 13; the outcomes of those 13 are presented in Table 2. Pathogens and contaminants, as determined by tissue sample (Atkins et al., 1998), are reported in Table 3. Fourteen results were available to compare the accuracy of blood culture and universal containers, the results of which are displayed in Table 4. Positive post-test probabilities of $\mathrm{CRP}(>5 \mathrm{mg} / \mathrm{L}), \mathrm{CRP}(>10.3 \mathrm{mg} / \mathrm{L})$ and ESR $(>13 \mathrm{~mm} / \mathrm{h})$ were $0.21,0.23$ and 0.15 respectively. The negative post-test probabilities of CRP $(<5 \mathrm{mg} / \mathrm{L})$, CRP $(<10.3 \mathrm{mg} / \mathrm{L})$ and ESR $(<13 \mathrm{~mm} / \mathrm{h})$ were $0.07,0.10$ and 0.14 respectively.

\subsection{Aspiration volume}

Aspiration volume for infected $(n=17)$ and non-infected $(n=108)$ joints was compared. The mean volume for infected and non-infected joints was $6 \mathrm{~mL}(2-36 \mathrm{~mL})$ and $11 \mathrm{~mL}(1-200 \mathrm{~mL})$ respectively. We ascertained that both in- 
Table 1. Statistical analysis of CRP, ESR and aspiration; biopsy included for reference.

\begin{tabular}{|c|c|c|c|c|c|}
\hline & $\begin{array}{r}\mathrm{CRP}>5 \mathrm{mg} / \mathrm{L} \\
(n=117)\end{array}$ & $\begin{array}{r}\mathrm{CRP}>10.3 \mathrm{mg} / \mathrm{L} \\
(n=117)\end{array}$ & $\begin{array}{r}\mathrm{ESR}>13 \mathrm{~mm} / \mathrm{h} \\
(n=117)\end{array}$ & $\begin{array}{r}\text { Aspiration } \\
(n=125)\end{array}$ & $\begin{array}{r}\text { Biopsy } \\
(n=125)\end{array}$ \\
\hline True positive & 13 & 9 & 12 & 10 & 17 \\
\hline True negative & 31 & 70 & 31 & 101 & 108 \\
\hline False positive & 69 & 30 & 69 & 7 & 0 \\
\hline False negative & 5 & 8 & 5 & 7 & 0 \\
\hline Sensitivity (\%) (95\% CI) & $76(56-97)$ & $53(29-76)$ & $71(49-92)$ & $59(35-82)$ & \\
\hline Specificity (\%) (95\% CI) & $51(41-61)$ & $70(61-79)$ & $31(22-40)$ & $94(89-98)$ & \\
\hline Pre-test probability & 0.15 & 0.15 & 0.15 & 0.14 & 0.14 \\
\hline Positive post-test probability & 0.21 & 0.23 & 0.15 & 0.59 & \\
\hline Negative post-test probability & 0.07 & 0.10 & 0.14 & 0.06 & \\
\hline Positive predictive value $(\%)(95 \% \mathrm{CI})$ & $21(11-31)$ & $23(10-36)$ & $15(7-23)$ & $59(35-82)$ & \\
\hline Negative predictive value $(\%)(95 \% \mathrm{CI})$ & $93(86-99)$ & $90(86-99)$ & $86(74-97)$ & $94(89-98)$ & \\
\hline
\end{tabular}

Table 2. Outcomes of patients receiving antibiotics at the time of revision.

\begin{tabular}{lr}
\hline Species & Number \\
\hline True positive & 1 \\
True negative & 10 \\
False positive & 2 \\
False negative & 0 \\
\hline
\end{tabular}

fected and non-infected groups did not display a normal distribution $(W=0.5573, p<0.001$ and $W=0.39955, p<$ 0.001 respectively). Hence, comparison of the two populations required a non-parametric test, which could not disprove that they did not come from the same distribution $(W=758, p=0.2458)$.

\subsection{Dry tap}

The five aspirations that were dry taps had $10 \mathrm{~mL}$ of normal saline infiltrated and then immediately aspirated. Two grew an organism ( $P$. aeruginosa, $S$. aureus), whereas three did not. Compared to tissue sampling, $P$. aeruginosa was cultured in three of five samples, and $S$. aureus was cultured in five of five samples. Of the three aspirates which were sterile, two had no positive tissue cultures and one was culture positive for $P$. acnes in one of five samples. According to our definition, all five aspirations agreed with the biopsy result.

\section{Discussion}

PJI diagnostics is a difficult clinical problem in which a range of diagnostic tests inform decision making. Our data suggest that a positive synovial fluid culture may well be erroneous (positive post-test probability 0.59 ) but that a negative sample is highly likely to be negative (negative post-test probability 0.06 ). In our population the pre-test probability of infection was 0.14 . These data suggest the following for our cohort: before an aspiration is performed, 14 out of 100 individuals truly have a PJI; after a positive aspirate, 59 in 100 individuals truly have a PJI and 41 in 100 do not. After a negative aspirate 6 in 100 do have an infection, whereas 94 in 100 individuals do not.

A recent review of serum biomarkers in PJI reported the sensitivity of CRP and ESR to range from $74 \%$ to $94 \%$ and from $42 \%$ to $94 \%$ respectively; specificity of CRP and ESR ranged from $20 \%$ to $100 \%$ and from $33 \%$ to $87 \%$ respectively (Saleh et al., 2018). The wide range of values above belies the difficulty in studying serological markers when assessing PJI. Our data are mostly in keeping with the previous literature, though in this cohort of patients the specificity of ESR was lower than other similar studies (Saleh et al., 2018).

We decided to compare the threshold for CRP from a meta-analysis (Piper et al., 2010) versus the common clinical value of $>5 \mathrm{mg} / \mathrm{L}$. Our data show that $<5 \mathrm{mg} / \mathrm{L}$ would be a more reliable value to rule out infection in our cohort. The data from our cohort indicate that $>5 \mathrm{mg} / \mathrm{L}$ is comparable to $>10.3 \mathrm{mg} / \mathrm{L}$ when positively identifying infection. We would state that neither CRP nor ESR are suitable as positive identifiers of infection, though CRP $<5 \mathrm{mg} / \mathrm{L}$ has a strong negative predictive value. A conclusion from our results is that CRP is a more useful blood marker than ESR as a sole indicator.

A recent large retrospective analysis offered that sensitivity of a dry tap is comparable to a normal aspiration, whereas specificity was lower (Partridge et al., 2018). This is in keeping with previous findings in a much smaller cohort (Ali et al., 2006). Of our five dry taps we determined that aspiration culture was in agreement with the tissue culture in each instance. One patient grew $P$. acnes in a single tissue sample; in that instance the patient's CRP was $1 \mathrm{mg} / \mathrm{L}$ and ESR $8 \mathrm{~mm} / \mathrm{h}$, suggesting that the single tissue sample was a contaminant. We would encourage future authors to report data on dry taps to increase the body of evidence.

We were unable to discern a difference in aspiration volume between the infected and non-infected groups. Fluid 
Table 3. Pathogens and contaminants. Pathogenic indicates that three or more tissue samples grew concordant species. Contaminants are cases where fewer than three tissue samples grew concordant species; this has been subdivided to show when a single or two tissue samples were culture positive.

\begin{tabular}{|c|c|c|c|}
\hline Species & Pathogenic instances & Contaminant (1) & Contaminant (2) \\
\hline Staphylococcus epidermidis & 7 & 5 & 1 \\
\hline Propionibacterium acnes & 3 & 4 & 2 \\
\hline Staphylococcus aureus & 2 & & \\
\hline Candida sp. & 1 & & \\
\hline Pseudomonas aeruginosa & 1 & & \\
\hline Staphylococcus capitis & 1 & & \\
\hline Staphylococcus lugdenensis & 1 & & \\
\hline Staphyloccus saccharolyticus & 1 & & \\
\hline Streptococcus milleri & & & 1 \\
\hline Staphylococcus warneri & & & 1 \\
\hline Enterococcus faecalis & & 1 & \\
\hline Coagulase negative Staphylococcus sp. & & 1 & \\
\hline Microccus luteus & & 1 & \\
\hline Staphylococcus cohnii & & 1 & \\
\hline Staphyloccus cohnii urealyticum & & 1 & \\
\hline
\end{tabular}

Table 4. Comparison of blood cultures with universal containers; 14 of 17 cases positive for PJI were available for comparison. In three cases no universal container was sent for analysis.

\begin{tabular}{lrr}
\hline & Blood culture bottle & Universal container \\
\hline True positive & 9 & 5 \\
True negative & 1 & 1 \\
False positive & 4 & 4 \\
False negative & 0 & 4 \\
\hline
\end{tabular}

volume difference was found to be not significant in a previous study (Ali et al., 2006), though again the cohort was small, and so we would encourage authors to report these data. Synovial fluid was collected in an aerobic, anaerobic and sterile universal container. We hypothesised that the sterile containers were less accurate, as reported in a previous study (Jordan et al., 2014). The appearance that universal containers are less accurate is in keeping with previous opinion within the laboratory. In the future our lab does not plan to use universal containers when aspirating a hip joint for periprosthetic joint infection.

Limitations of this study include not considering patient factors such as age, gender, disease state, medication state or type of implant. There is a methodological limitation in some, but not all specimens were cultured for $14 \mathrm{~d}$, which may lower detection of slow-growing bacteria such as $P$. $a c$ nes (Schwotzer et al., 2014); given P. acnes was the most common pathogen and contaminant in this study, this may be a point for further study. Setting tissue sampling as a control test is recognised as a limitation of the study due to the risk of culture-negative infections, a recognised phenomenon (Tan et al., 2018).
In this study we used thresholds for CRP derived from a meta-analysis (Piper et al., 2010) and common clinical practice. If we were looking for a rule-out test, then CRP at a threshold of $5 \mathrm{mg} / \mathrm{L}$ is most useful. This competes very effectively with aspiration culture, although aspiration has other opportunities for analysis, including white cell count and differential, alphadefensin, and synovial CRP, DNA analysis and others (Dinneen et al., 2013; Bingham et al., 2014; Bonanzinga et al., 2017; Gollwitzer et al., 2013; Gallo et al., 2018; Tetreault et al., 2014; Gomez et al., 2012). Given the increased treatment burden of aspiration, the authors only recommend its use in association with other synovial tests.

Ethical statement. This service evaluation was conducted in the Northumbria Healthcare NHS Foundation Trust. Ethical approval deemed not required by the Health Research Authority.

Code availability. The code used for analysing aspiration volume distributions is available on request.

Data availability. The data used for this article are available on reasonable request.

Author contributions. CJB provided investigation, formal analysis and writing of the manuscript. AM provided resources and investigation and also conceptualisation. MK provided supervision and a methodology. TO provided resources and supervision. SJT supported investigation. PFP supported supervision and supplied resources. IC supported supervision and supplied resources. MRR 
provided conceptualisation, methodology, project administration, resources, supervision and then reviewed and edited the manuscript.

Competing interests. The authors declare that they have no conflict of interest.

Review statement. This paper was edited by Alex McLaren and reviewed by three anonymous referees.

\section{References}

Ali, F., Wilkinson, J. M., Cooper, J. R., Kerry, R. M., Hamer, A. J., Norman, P., and Stockley, I.: Accuracy of joint aspiration for the preoperative diagnosis of infection in total hip arthroplasty, J. Arthroplasty, 21, 221-226, https://doi.org/10.1016/j.arth.2005.05.027, 2006.

Atkins, B. L., Athanasou, N., Deeks, J. J., Crook, D. W. M., Simpson, H., Peto, T. E. A., McLardy-Smith, P., Berendt, A. R., and Group, T. O. C. S.: Prospective Evaluation of Criteria for Microbiological Diagnosis of Prosthetic-Joint Infection at Revision Arthroplasty, J. Clin. Microbiol., 36, 2932-2939, 1998.

Bingham, J., Clarke, H., Spangehl, M., Schwartz, A., Beauchamp, C., and Goldberg, B.: The alpha defensin-1 biomarker assay can be used to evaluate the potentially infected total joint arthroplasty, Clin. Orthop. Relat. R., 472, 4006-4009, https://doi.org/10.1007/s11999-014-3900-7, 2014.

Bonanzinga, T., Zahar, A., Dütsch, M., Lausmann, C., Kendoff, D., and Gehrke, T.: How Reliable Is the Alpha-defensin Immunoassay Test for Diagnosing Periprosthetic Joint Infection? A Prospective Study, Clin. Orthop. Relat. R., 475, 408-415, https://doi.org/10.1007/s11999-016-4906-0, 2017.

Cross, M. C., Kransdorf, M. J., Chivers, F. S., Lorans, R., Roberts, C. C., Schwartz, A. J., and Beauchamp, C. P.: Utility of percutaneous joint aspiration and synovial biopsy in identifying culturepositive infected hip arthroplasty, Skeletal Radiol., 43, 165-168, https://doi.org/10.1007/s00256-013-1757-6, 2014.

Dinneen, A., Guyot, A., Clements, J., and Bradley, N.: Synovial fluid white cell and differential count in the diagnosis or exclusion of prosthetic joint infection, Bone Joint J., 95-B, 554-557, https://doi.org/10.1302/0301-620x.95b4.30388, 2013.

Gallo, J., Svoboda, M., Zapletalova, J., Proskova, J., and Juranova, J.: Serum IL-6 in combination with synovial IL6/CRP shows excellent diagnostic power to detect hip and knee prosthetic joint infection, PLoS ONE, 13, e0199226, https://doi.org/10.1371/journal.pone.0199226, 2018.

Gollwitzer, H., Dombrowski, Y., Prodinger, P. M., Peric, M., Summer, B., Hapfelmeier, A., Saldamli, B., Pankow, F., von Eisenhart-Rothe, R., Imhoff, A. B., Schauber, J., Thomas, P., Burgkart, R., and Banke, I. J.: Antimicrobial peptides and proinflammatory cytokines in periprosthetic joint infection, J. Bone Joint Surg. Am., 95, 644-651, 2013.

Gomez, E., Cazanave, C., Cunningham, S., Quaintance, K., Steckelberg, J., Uhl, J., Hanssen, A., Karau, M., Schmidt-Malan, S., Osmon, D., Berbari, E., Mandrekar, J., and Patel, R.: Prosthetic Joint Infection Diagnosis Using Broad-Range PCR of Biofilms Dislodged from Knee and Hip Arthroplasty Sur- faces Using Sonication, J. Clin. Microbiol., 50, 3501-3508, https://doi.org/10.1128/JCM.00834-12, 2012.

Jordan, R. W., Smith, N. A., Saithna, A., Sprowson, A. P., and Foguet, P.: Sensitivities, Specificities, and Predictive Values of Microbiological Culture Techniques for the Diagnosis of Prosthetic Joint Infection, BioMed Research International, 2014, 5, https://doi.org/10.1155/2014/180416, 2014.

Partridge, D. G., Winnard, C., Townsend, R., Cooper, R., and Stockley, I.: Joint aspiration, including culture of reaspirated saline after a "dry tap", is sensitive and specific for the diagnosis of hip and knee prosthetic joint infection, Bone Joint J., 100-B, 749-754, https://doi.org/10.1302/0301-620X.100B6.BJJ-20170970.R2, 2018.

Parvizi, J., Jacovides, C., Zmistowski, B., and Jung, K. A.: Definition of periprosthetic joint infection: is there a consensus?, Clin. Orthop., 469, 3022-3030, https://doi.org/10.1007/s11999011-1971-2, 2011.

Parvizi, J., Gehrke, T., and Chen, A. F.: Proceedings of the International Consensus on Periprosthetic Joint Infection, Bone Joint J., 95-B, 1450-1452, https://doi.org/10.1302/0301620X.95B11.33135, 2013.

Parvizi, J., Tan, T. L., Goswami, K., Higuera, C., Della Valle, C., Chen, A. F., and Shohat, N.: The 2018 Definition of Periprosthetic Hip and Knee Infection: An EvidenceBased and Validated Criteria, J. Arthroplasty, 33, 1309-1314, https://doi.org/10.1016/j.arth.2018.02.078, 2018.

Peel, T. N., Dowsey, M. M., Buising, K. L., Liew, D., and Choong, P. F. M.: Cost analysis of debridement and retention for management of prosthetic joint infection, Clin. Microbiol. Infec., 19, 181-186, https://doi.org/10.1111/j.1469-0691.2011.03758.x, 2013.

Piper, K. E., Fernandez-Sampedro, M., Steckelberg, K. E., Mandrekar, J. N., Karau, M. J., Steckelberg, J. M., Berbari, E. F., Osmon, D. R., Hanssen, A. D., Lewallen, D. G., Cofield, R. H., Sperling, J. W., Sanchez-Sotelo, J., Huddleston, P. M., Dekutoski, M. B., Yaszemski, M., Currier, B., and Patel, R.: C-reactive protein, erythrocyte sedimentation rate and orthopedic implant infection, PLoS ONE, 5, e9358, https://doi.org/10.1371/journal.pone.0009358, 2010.

Public Health England: B $44 \mathrm{i} 2$ Investigation of orthopaedic implant associated infections, available at: https://www.gov.uk/government/publications/ smi-b-44-investigation-of-prosthetic-joint-infection-samples (last access: 14 February 2021), Public Health England, 21-22, 2016.

RStudio: Integrated Development for R, RStudio, Inc., Boston, MA, available at: http://www.rstudio.com (last access: 14 February 2021), 2015.

Saleh, A., George, J., Faour, M., Klika, A. K., and Higuera, C. A.: Serum biomarkers in periprosthetic joint infections, Bone Joint Res., 7, 85-93, https://doi.org/10.1302/2046-3758.71.BJR-20170323, 2018.

Schwotzer, N., Wahl, P., Fracheboud, D., Gautier, E., and Chuard, C.: Optimal culture incubation time in orthopedic device-associated infections: a retrospective analysis of prolonged 14-day incubation, J. Clin. Microbiol., 52, 61-66, https://doi.org/10.1128/JCM.01766-13, 2014.

Tan, T. L., Kheir, M. M., Shohat, N., Tan, D. D., Kheir, M., Chen, C., and Parvizi, J.: Culture-Negative Periprosthetic Joint Infection: 
An Update on What to Expect, JBJS Open Access, 3, e0060, 2018.

Tanner, J., Padley, W., Kiernan, M., Leaper, D., Norrie, P., and Baggott, R.: A benchmark too far: findings from a national survey of surgical site infection surveillance, J. Hosp. Infect., 83, 87-91, https://doi.org/10.1016/j.jhin.2012.11.010, 2013.

Tetreault, M., Wetters, N., Moric, M., Gross, C., and Della Valle, C.: Is Synovial C-reactive Protein a Useful Marker for Periprosthetic Joint Infection?, Clin. Orthop. Relat. R., 472, 3997-4003, https://doi.org/10.1007/s11999-014-3828-y, 2014.
Tingle, S. J., Marriott, A., Partington, P. F., Carluke, I., and Reed, M. R.: Performance and learning curve of a surgical care practitioner in completing hip aspirations, Ann. R. Coll. Surg. Engl., 98, 543546, https://doi.org/10.1308/rcsann.2016.0315, 2016. 